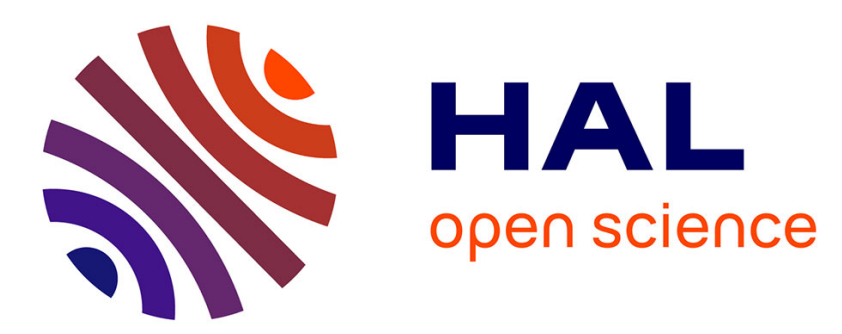

\title{
Les études chinoises et la professionnalisation : la filière LEA a-t-elle un avenir?
}

Lu Shi

\section{To cite this version:}

Lu Shi. Les études chinoises et la professionnalisation : la filière LEA a-t-elle un avenir?. Association Française d'Etudes chinoises. Etudier et enseigner la Chine, Association Française d'Etudes chinoises, pp.103-118, 2010. halshs-00577752

\section{HAL Id: halshs-00577752 https://shs.hal.science/halshs-00577752}

Submitted on 17 Mar 2011

HAL is a multi-disciplinary open access archive for the deposit and dissemination of scientific research documents, whether they are published or not. The documents may come from teaching and research institutions in France or abroad, or from public or private research centers.
L'archive ouverte pluridisciplinaire $\mathbf{H A L}$, est destinée au dépôt et à la diffusion de documents scientifiques de niveau recherche, publiés ou non, émanant des établissements d'enseignement et de recherche français ou étrangers, des laboratoires publics ou privés. 


\section{Les études chinoises et la professionnalisation : la filière LEA a-t-elle un avenir ?}

\section{Shi Lu}

Au cours des dernières décennies, l'un des changements les plus importants dans l'enseignement supérieur en France est sans doute le mouvement de professionnalisation des formations. L'Université, longtemps considérée comme lieu de transmission de savoir, se transforme en lieu de transmission de savoir-faire. L'accès des jeunes à l'emploi devient l'un des principaux soucis, sinon une priorité. Ainsi les formations professionnelles se multiplient-elles aussi bien dans les filières techniques que dans les formations classiques en sciences humaines et sociales. Rapprocher le système éducatif du monde du travail et répondre aux besoins du marché sont autant de raisons évoquées pour expliquer l'ampleur de ce mouvement et justifier la politique de professionnalisation des enseignements universitaires.

La professionnalisation des études supérieures repose sur deux grands axes. D'une part, on crée des écoles spécialisées pour un secteur d'activités bien définies, et d'autre part, on réforme les universités pour donner une dimension professionnelle à toutes les filières. C'est bien ce dernier cas qui nous intéresse en particulier. Ici, nous partons plus précisément d'observations sur les études chinoises dans la filière LEA (Langues étrangères appliquées) pour réfléchir sur la professionnalisation. Les études chinoises ont été longtemps réservées à la formation de spécialistes de la Chine ancienne, moderne et contemporaine en histoire, philosophie, littérature ou linguistique. Depuis les années 1980, à la suite de l'ouverture économique de la Chine, l'enseignement du chinois dans les universités françaises s'est tourné davantage vers de nouveaux horizons : généralisation de l'enseignement du chinois pour le grand public (formation continue, cours 


\section{Shi Lu}

optionnel pour les étudiants non-spécialistes, etc.) et développement de la filière LEA.

Cette dernière est appréciée par certains comme une voie d'accès au marché chinois désormais incontournable, mal-aimée par d'autres et considérée comme une vulgarisation de la sinologie. Aujourd'hui, plus de trente ans après la création de cette filière, quel bilan pouvons-nous dresser? Cette filière forme-t-elle vraiment les spécialistes du monde économique chinois ? Pour répondre à ces questions, nous reviendrons sur le mouvement de professionnalisation des formations universitaires, puis nous nous focaliserons sur la place des études chinoises dans la formation LEA. L'observation des pratiques d'enseignement dans les LEA anglaischinois nous aidera à dessiner des perspectives dans le contexte des mutations économiques et sociales que nous traversons.

\section{La professionnalisation des formations universitaires}

Longtemps, on a considéré que les missions principales et le rôle premier de l'Université consistaient à transmettre les connaissances. Mais progressivement, la question de la professionnalisation de l'enseignement supérieur est devenue un enjeu essentiel. Elle concerne l'articulation entre savoirs théoriques et pratiques, les rapports entre universités et entreprises et le lien entre formation et accès à l'emploi. Les dernières décennies ont été marquées par une diversification des filières professionnelles dans l'enseignement supérieur. La conciliation entre le monde du travail et le monde éducatif constitue ainsi une politique délibérée. Les universités s'adaptent à ces changements et préparent les étudiants à s'insérer dans les activités économiques et sociales.

Qu'entendons-nous par professionnalisation ? La formation professionnelle est en général définie comme une formation qui rend apte à exercer une activité économique déterminée avec une connaissance précise et qui a pour finalité l'insertion professionnelle ${ }^{1}$. On l'appelle également

1 Jean Vincens et Sylvère Chirache, Rapport de la commission Professionnalisation des enseignements supérieurs, Paris : Haut comité Éducation et Économie, 1992 ; François Goulard, L'Enseignement supérieur en France, état des lieux et propositions, ministère de l'Enseignement supérieur et de la recherche, 2007 (http://lesrapports.ladocumentationfrancaise.fr/BRP/074000710/0000.pdf). 


\section{Les études chinoises et la professionnalisation}

formation professionnalisée. Certains spécialistes de l'éducation pensent cependant que la professionnalisation de l'enseignement supérieur peut concerner aussi une formation non étroitement spécialisée, mais destinée à préparer à la vie active en garantissant la transversalité des acquis et en développant les capacités d'adaptation des personnes, combinant formation théorique et apprentissage en situation de travail ${ }^{2}$. De ce fait, on parle aussi de la formation pré-professionnelle qui conduit à une large gamme d'emplois en combinant les capacités transversales et aussi les compétences dans une discipline donnée ${ }^{3}$.

La professionnalisation se développe avec l'élargissement et la diversification des formations professionnelles. Nous pouvons distinguer deux types de professionnalisation dans l'enseignement supérieur : professionnalisation des filières spécialisées et professionnalisation des enseignements dans les universités. Du fait que le système éducatif français distingue les grandes écoles et les universités, le mouvement de la professionnalisation suit des rythmes différents. La professionnalisation de l'enseignement supérieur s'est limitée au début à des formations spécifiques et techniques dans les grandes écoles : écoles d'ingénieurs, écoles de commerce et écoles de gestion, etc. Ces formations préparent les élèves à un métier précis. La professionnalisation des enseignements dans les universités est advenue plus tardivement, mais a largement gagné en ampleur au cours des trente dernières années.

\section{La professionnalisation des enseignements dans les universités}

La professionnalisation des enseignements dans les universités a commencé par les filières spécifiques et techniques dans les années 1960 avec la création des Institut universitaires de technologie (IUT), puis des Diplômes d'études supérieures spécialisées (DESS) en 1974, les Instituts universitaires de formation des maîtres (IUFM) en 1989 et les Instituts universitaires professionnels (IUP) en 1991, qui sont remplacés en 1999 par les licences professionnelles. Dans un premier temps, la professionnalisation concerne surtout les filières techniques et spécialisées. Le nombre d'inscrits dans ces filières s'est considérablement accru. Entre 1980 et 2000, le nombre d'étudiants inscrits en

2 François Goulard, opus cit., p. 51.

3 Idem. 


\section{Shi Lu}

IUT, une formation courte, a doublé, passant de 53700 à 119200 en $2000^{4}$. Ce succès entraîne une généralisation de la professionnalisation dans les universités. Désormais, toutes les filières universitaires doivent avoir une mission d'enseignement professionnel. Il s'agit de réformer les filières universitaires afin de rendre les étudiants aptes à intégrer une activité économique. C'est donc dans le contexte d'une politique nationale que la professionnalisation commence à toucher les cursus classiques, même les filières qui répondent difficilement aux critères de la professionnalisation.

\section{La professionnalisation des enseignements de langues}

La création d'une filière professionnelle se situe bien souvent en continuité d'une formation déjà existante. Dans les études de langues, la création en 1973 de la filière LEA est une bifurcation de la filière LLCE (Langues, littératures et civilisations étrangères). D'une part, elle répond à une demande du marché et des étudiants qui, attirés par l'étude des langues étrangères, n'envisagent pas de s'orienter vers les métiers de l'enseignement ou de la recherche, mais plutôt vers les entreprises, les administrations, les organisations ou les associations. D'autre part, elle constitue surtout une alternative à la filière LLCE, une formation académique souvent insuffisante pour faire des étudiants de bons traducteurs ou interprètes, et n'offrant pas assez de débouchés dans l'enseignement et la recherche. L'espoir a été mis sur la filière LEA pour rendre les études de langues professionnalisantes. Cette nouvelle filière se fixe pour objectif de former des cadres d'entreprises et d'administration dans le cadre d'un diplôme Bac +4 . Elle met en avant les compétences dans deux langues avec un volet de formation complémentaire en général en économie, droit et marketing. Le LEA est considéré comme une formation pluridisciplinaire qui prépare les diplômés à exercer un emploi dans les secteurs du commerce international, de la communication interculturelle, du tourisme, de la logistique et de la traduction.

Cette filière pré-professionnelle semble attractive, puisque depuis 1995, les effectifs en LEA se sont maintenus autour de 35000 dans plus de

\footnotetext{
4 José Rose, «La professionnalisation des études supérieures : tendances, acteurs et formes concrètes » in «Les chemins de la formation vers l'emploi : première biennale formation-emploi-travail », Relief, 2008-25, p. 46, http://portail.cereq.fr/ docenligne/2008/relief25-rose.pdf.
} 


\section{Les études chinoises et la professionnalisation}

cinquante universités françaises ${ }^{5}$. Plusieurs enquêtes et études confirment que la filière permet aux étudiants de s'intégrer rapidement au le marché du travail, essentiellement dans des fonctions commerciales ou de gestion administrative ${ }^{6}$. L'enquête réalisée par le Service commun universitaire d'information et d'orientation de l'université Lyon 3 montre qu'à l'issue du master II professionnel dont 86 \% sont des diplômés de LEA - toutes langues confondues -, $72 \%$ d'entre eux ont un emploi ; ils sont à $80 \% 18$ mois après leur diplôme ${ }^{7}$.

Le développement des formations professionnelles a été guidé par une volonté politique. La création en 1999 des licences professionnelles s'est inscrite dans le processus de continuité de la politique de professionnalisation. Lors de la rentrée 2000, 178 licences professionnelles ont été créées ${ }^{8}$. La professionnalisation dans des universités a aussi été initiée par les pouvoirs politiques régionaux. L'implication des collectivités locales dans l'enseignement supérieur a largement contribué à une diversification de l'offre. Les régions apportent des aides à la création de formations. Par une politique plus volontariste, elles peuvent aussi structurer les politiques, fixer des objectifs et susciter des demandes des organismes de formation en fonction des besoins spécifiques locaux ${ }^{9}$. Enfin, le développement des formations professionnelles est aussi venu des établissements universitaires et

5 Philippe Jacqué, " Même l'anglais ne remplit pas les amphis », Le Monde, dossier éducation, 10 février 2010, p. 7.

${ }^{6}$ Observatoire des formations, de l'insertion professionnelle et de la vie étudiante, Le Devenir des diplômés de licence, promotion 2005/2006, la Rochelle : université de la Rochelle, 2009 ; Agence pour l'emploi des cadre (APEC), L'Insertion professionnelle des jeunes diplômés en 2003, octobre 2003 ; Service commun universitaire d'information et d'orientation (SCUIO) de Lyon 3, L'Insertion professionnelle des étudiants inscrits en master 2 professionnel-Faculté des langues, 2007.

7 SCUIO Lyon 3, opus cit.

8 Jean-François Giret, "Le mouvement de professionnalisation des études supérieures, L'exemple des licences professionnelles », in " Les chemins de la formation vers l'emploi : première biennale formation-emploi-travail », Relief, 2008-25, p. 59-62.

9 Laure Gayraud, «Professionnalisation dans l'enseignement supérieur : quelles logiques territoriales ?», Net.doc. 59, http://www.cereq.fr/pdf/Net-Doc-59.pdf. 
des entreprises qui se sont beaucoup rapprochées. Dans la deuxième partie de notre réflexion, nous revenons, à travers le cas des études chinoises dans la formation LEA, sur l'adaptation d'une filière de langue à la professionnalisation.

\section{Les études chinoises et le LEA : quelles spécificités et quel avenir?}

Dans les années 1980, le marché chinois s'ouvre et la Chine devient un eldorado pour les affaires. La formation des spécialistes de la Chine se diversifie pour s'adapter au nouveau contexte. D'une part, l'enseignement du chinois se démocratise et devient accessible pour un public non-spécialiste, et d'autre part, la filière LEA est créée dans des universités pour mieux répondre à la demande du marché chinois et des étudiants. Cette filière se veut pragmatique et tournée vers les entreprises en lien avec le monde chinois. Soucieux de leur insertion professionnelle, les étudiants choisissent cette formation dans l'espoir de travailler plus tard avec le monde chinois. Le LEA vise ainsi dès son départ à former des employés de rang intermédiaire pour les diplômés de la licence $(\mathrm{Bac}+3)$ et de rang cadre pour les diplômés Bac +4 dans des métiers divers : tourisme, importexport, marketing et gestion, etc. Trente ans plus tard, la formation est-elle à la hauteur de ses ambitions d'origine ?

\section{Le LEA anglais-chinois en France}

La toute première filière LEA anglais-chinois aurait vu le jour en 1973 à l'université de Bordeaux 3, puis quelques années plus tard à l'université d'Aix-en-Provence. Jusqu'alors, les études chinoises avaient été réservées à la formation de spécialistes de la Chine dans le cadre de la filière classique LLCE. Il faut attendre la décennie 1990 pour voir se multiplier les formations LEA anglais-chinois ; le mouvement s'accélère dans les années 2000 - avec notamment trois nouvelles habilitations de licence pour la seule année 2009 : Metz, Grenoble et Brest.

Nous recensons aujourd'hui seize formations LEA anglais-chinois en France ${ }^{10}$. Elles se répartissent un peu partout sur l'hexagone. Les formations

10 Les informations proviennent de deux sources : le site Internet de l'Association 


\section{Les études chinoises et la professionnalisation}

LEA et LLCE coexistent dans six universités. En comparaison avec la filière LEA, seulement huit établissements d'enseignement supérieur dispensent la formation LLCE chinois ${ }^{11}$. Cet écart s'explique par les difficultés de débouchés pour les linguistes et les littéraires. Les étudiants affluent vers la filière LEA. Les effectifs du LEA augmentent et dépassent de loin la filière classique LLCE. À l'université Jean Moulin Lyon 3, depuis de nombreuses années, les inscrits en première année de licence LEA sont deux fois plus nombreux qu'en LLCE.

Les enseignements en licence LEA sont fondés essentiellement sur deux langues étrangères, les cultures qui leur sont associées et un volet de connaissances dites professionnelles en économie, droit et gestion des entreprises. La majorité des formations LEA anglais-chinois se focalisent sur deux types de formation au niveau de la licence : affaires et commerce et traduction. Cependant, une diversité de parcours est offerte à partir de la seconde année de licence (L2) et notamment en troisième année (L3), tels que le tourisme, le droit des affaires internationales, la traduction spécialisée et ainsi de suite. C'est le cas à Bordeaux 3, Toulouse le Mirail, Montpellier 3 et Aix-en-Provence. Néanmoins, ces parcours professionnels sont en général communs à toutes les langues et les spécificités géoculturelles de la langue du pays étudiée ne sont pas privilégiées. Seule l'université de la Rochelle offre une formation LEA ciblée sur une aire géoculturelle : l'Asie Pacifique (Chine, Corée, Indonésie, Singapour, Hong Kong, Australie et Nouvelle-Zélande). Deux parcours sont proposés dans cette spécialité: «Langues, cultures et affaires », parcours renforcé avec plus de langue appliquée et de civilisation, et « Langues et économie » avec une dominante d'économie appliquée. Dans le premier parcours, les étudiants se dirigent vers deux directions à partir de licence 2 : la découverte d'une deuxième langue asiatique ou une préparation en économie pour les étudiants qui envisagent d'emprunter le parcours « Langues et économie » ouvert en licence 3 .

nationale de langues étrangères appliquées (http://anlea.org) et les sites Internet des universités concernées.

11 À part les six universités qui figurent sur la carte avec le sigle de LLCE, l'INALCO et l'université de Lille 3 proposent également la formation LLCE. 
Figure 1 : Formations licence LEA anglais-chinois en France ${ }^{12}$

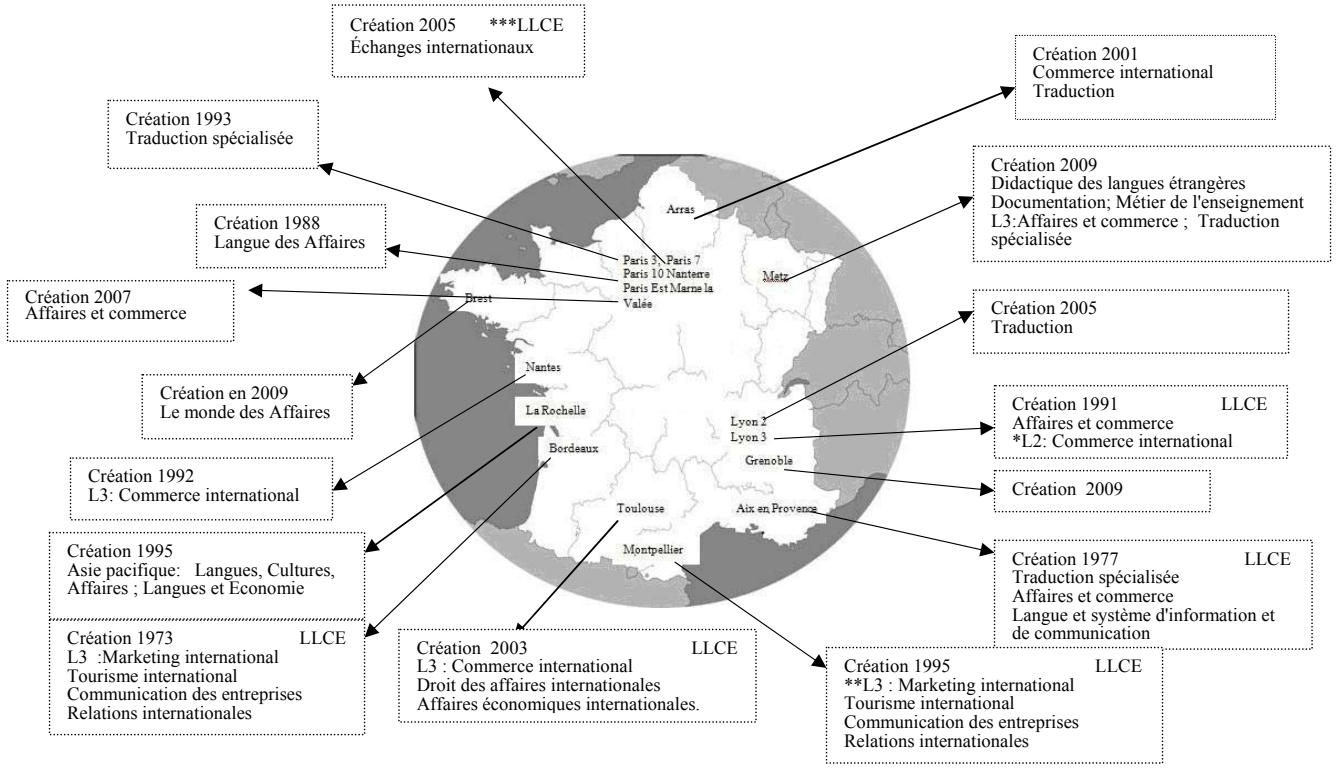

*L2 : licence $2, * *$ L3 $:$ licence $3, * * *$ LLCE $:$ la filière LLCE dans certaines universités

C'est à partir du master que les étudiants choisissent leur spécialisation dans un domaine allant du management et des négociations interculturelles aux marchés émergents en passant par la traduction spécialisée et le commerce international.

Le tableau 1 illustre la multitude de mentions dans la formation master LEA. Comme en licence, les universités disposent d'une certaine autonomie pour définir les parcours et les spécialisations en fonction des spécificités et des compétences pédagogiques de l'établissement et jouer de la concurrence. De ce fait, les maquettes d'enseignements sont très variables. La plupart des spécialisations commencent en master 1, rares sont les universités qui proposent une ou des spécialisations en dernière année de master. Certaines formations sont la poursuite naturelle de la licence LEA, d'autres sont plus sélectives et limitent le nombre de places. À Lyon 3, les spécialisations de master 1 Langues-droit-gestion et Communication internationale des entre-

12 Nous remercions Véronique Alexandre-Journeau d'avoir mis à notre disposition le fond de carte. 


\section{Les études chinoises et la professionnalisation}

prises et des administrations sont ouvertes à tous les étudiants ayant obtenu une licence LEA ou un équivalent, mais deviennent sélectives en master 2, comme le parcours Commerce international. Ce dernier est un parcours sélectif dès la seconde année de la licence.

En outre, si nous nous fions aux programmes de ces masters, peu de formations sont véritablement spécialisées sur le monde chinois. Le master Asie Pacifique de l'université de la Rochelle fait exception, puisque la formation en licence axée sur les pays asiatiques et océaniques se poursuit en master $^{13}$.

Tableau 1 : Formations master LEA anglais-chinois

\begin{tabular}{|l|l|}
\hline \multicolumn{1}{|c|}{ Université } & \multicolumn{1}{c|}{ Mentions } \\
\hline Artois & Négociations interculturelles \\
\hline Michel de Montaigne-Bordeaux 3 & Management et négociations interculturels \\
\hline La Rochelle & $\begin{array}{l}\text { Asie Pacifique: Langues, cultures, Affaires } \\
\text { Langues et économie }\end{array}$ \\
\hline Jean Moulin-Lyon 3 & $\begin{array}{l}\text { Communication internationale des entreprises } \\
\text { et des administrations } \\
\text { Commerce international } \\
\text { Langues-droit-gestion }\end{array}$ \\
\hline Provence Aix-Marseille 1 & $\begin{array}{l}\text { Affaires internationales et information stratégique } \\
\text { Métiers de la traduction }\end{array}$ \\
\hline Paul Valéry-Montpellier 3 & $\begin{array}{l}\text { Négociation de projets internationaux } \\
\text { Traduction }\end{array}$ \\
\hline Nantes & $\begin{array}{l}\text { Logistique internationale M2 } \\
\text { Commerce international }\end{array}$ \\
\hline Paris Ouest Nanterre La Défense & Management international et interculturel \\
\hline Sorbonne Nouvelle-Paris 3 & $\begin{array}{l}\text { Langues et affaires économiques } \\
\text { internationales } \\
\text { Traduction et terminologie juridique et } \\
\text { financière }\end{array}$ \\
\hline
\end{tabular}

13 Voir la contribution de Martine Raibaud dans ce volume, «L'adaptation de la sinologie dans une université nouvelle : l'exemple rochelais ». 


\section{Shi Lu}

\begin{tabular}{|l|l|}
\hline Paris Diderot & $\begin{array}{l}\text { Industrie de la langue et traduction spécialisée } \\
\text { M2 } \\
\text { Conception de documentation multilingue et } \\
\text { multimédia } \\
\text { Études interculturelles M2 }\end{array}$ \\
\hline Toulouse-Le Mirail & $\begin{array}{l}\text { Commerce international } \\
\text { Affaires économiques internationales } \\
\text { Stratégies juridiques du développement } \\
\text { international M2 }\end{array}$ \\
\hline
\end{tabular}

Cet aperçu de l'ensemble des LEA anglais-chinois invite à envisager plus en détail la place des études chinoises dans la formation. Comment est conçu l'enseignement du chinois dans la filière ? En quoi est-il différent de la filière LLCE ?

\section{L'enseignement du chinois dans la formation LEA}

En termes de pédagogie, l'enseignement du chinois en licence LEA suit le même modèle que la filière LLCE; on retrouve les cours classiques de grammaire, thème, version et pratique orale. Certaines universités, telle Bordeaux 3, ont des cours de langue communs aux deux filières. Compte tenu de la spécificité de la langue chinoise qui implique un long apprentissage des caractères, la plupart des formations LEA comme LLCE proposent des volumes horaires renforcés en langue pour les grands débutants en première année. Cette cohérence des enseignements de langue entre les deux filières, en première année notamment, est inscrite dans le cadre de l'orientation active. Elle permet aux étudiants de passer éventuellement d'une filière à l'autre - le plus souvent du LEA vers le LLCE - à la fin du premier semestre ou à la fin de la première année universitaire. C'est surtout à partir de la deuxième année que les enseignements de la langue se différencient entre les deux filières, c'est du moins le cas à Jean Moulin Lyon 3.

La comparaison des volumes horaires de langue et civilisation chinoises dans les deux filières révèle un grand écart entre les deux filières : au total 551 heures en LEA contre 815 heures en LLCE au cours des trois années de licence. L'enseignement de la langue proprement dite est quasi équilibré (30 heures de moins en LEA sur trois ans) ; c'est dans les matières de civilisation et de culture que les différences sont marquées. De manière générale, les cours de civilisation sont faiblement dotés en contenu et en volume horaire dans les 


\section{Les études chinoises et la professionnalisation}

formations LEA. À Lyon 3, au cours de la licence LEA, seulement 24 heures en première année (semestres 1 et 2 ) sont consacrées à l'histoire contemporaine de la Chine (culture et société) et 24 heures en troisième année (semestres 5 et 6) au système économique chinois (économie et entreprise). Alors que les étudiants en LLCE bénéficient de 108 heures d'enseignements pour l'histoire de la Chine (histoire culturelle) pendant tout leur cursus.

Certains intitulés ne sont pas sans ambiguïté ; ils peuvent être focalisés sur l'apprentissage de la langue ou de la culture comme « Langue et société » en LLCE, ou tournés plus ou moins vers un enseignement spécifique en lien avec l'apprentissage de la langue, tel est le cas de « l'Informatique spécifique » dans les deux filières. Faute de directives, les enseignants décident en fonction de leurs propres compétences.

Les variations sont également importantes d'un établissement à l'autre. À l'université d'Artois, l'enseignement en LEA consacré à la civilisation et l'histoire de la Chine va de la Chine ancienne à la Chine contemporaine, au rythme de 4 heures hebdomadaires pendant les deux premières années de la licence, puis d'un cours thématique axé sur la société d'aujourd'hui (écologie, sécurité alimentaire, littérature et rencontres Chine-Occident) en licence 3 avec 2,5 heures hebdomadaires. Cela représente 273 heures sur trois ans, soit un volume horaire presque six fois plus important que celui de Lyon 3. Ces différences entre établissements pourraient s'expliquer en partie par les modalités de gestion des diplômes. Dans le cas de l'université Jean Moulin Lyon 3, une maquette unique des enseignements en LEA est imposée à toutes les langues; les langues rares comme le chinois ne bénéficient pas de souplesse de gestion. 


\section{Shi Lu}

Tableau 2 : cours de langue et civilisation chinoise en licence LLCE et LEA à l'université Jean Moulin Lyon 3

\begin{tabular}{|c|c|c|c|}
\hline \multicolumn{2}{|l|}{ Licence LLCE } & \multicolumn{2}{|c|}{ Licence LEA } \\
\hline Matières & $\begin{array}{l}\text { Nombre } \\
\text { d'heures }\end{array}$ & Matières & $\begin{array}{l}\text { Nombre } \\
\text { d'heures }\end{array}$ \\
\hline Stage débutants S1 & 50 & Stage débutants S1 & 50 \\
\hline $\begin{array}{l}\text { Grammaire CM } \\
\text { S1-S6 }\end{array}$ & 126 & $\begin{array}{l}\text { Grammaire CM } \\
\text { S1-S6 }\end{array}$ & 96 \\
\hline Thème TD S1-S6 & 90 & Thème TD S1-S6 & 90 \\
\hline Version TD S1-S6 & 90 & Version TD S1-S6 & 90 \\
\hline $\begin{array}{l}\text { Pratique orale TD } \\
\text { S1-S6 }\end{array}$ & 90 & $\begin{array}{l}\text { Pratique orale TD } \\
\text { S1-S6 }\end{array}$ & 90 \\
\hline $\begin{array}{ll}\text { Informatique } & \text { spécifique } \\
\text { TD S3-S4 } & \\
\end{array}$ & 35 & $\begin{array}{l}\text { Informatique } \\
\text { spécifique TD S4 }\end{array}$ & 15 \\
\hline Écriture CM S1 & 48 & $\begin{array}{l}\text { Langue et affaires CM } \\
\text { S3-S6 }\end{array}$ & 72 \\
\hline Documentation TD S1 & 10 & $\begin{array}{l}\text { Culture et société } \\
\text { moderne CM S1-S2 }\end{array}$ & 24 \\
\hline Études de textes CM S2 & 24 & $\begin{array}{l}\text { Économie et } \\
\text { entreprise CM S5-S6 }\end{array}$ & 24 \\
\hline $\begin{array}{l}\text { Histoire culturelle CM S2- } \\
\text { S6 }\end{array}$ & 108 & Total & 551 \\
\hline Littérature CM S3-S6 & 96 & & \\
\hline $\begin{array}{ll}\text { Informatique } & \text { spécifique } \\
\text { TD S3-S4 } & \\
\end{array}$ & 35 & & \\
\hline $\begin{array}{l}\text { Langue de communication } \\
\text { CM S3-S4 }\end{array}$ & 24 & & \\
\hline $\begin{array}{l}\text { Langue et société CM S3- } \\
\text { S6 }\end{array}$ & 24 & & \\
\hline Total & 815 & & \\
\hline
\end{tabular}

Note : S1 à S6 désignent les 6 semestres de la licence, TD les travaux dirigés et CM les cours magistraux.

Au niveau du master, le volume horaire de langue chinoise se réduit à six heures hebdomadaires en moyenne avec peu d'écarts d'une université à l'autre. L'enseignement se spécialise : langue juridique, la traduction technique, etc. Dans toutes les formations, les étudiants effectuent en outre un stage linguistique ou professionnel dans le monde sinisé.

La formation LEA reste en fait très généraliste et ne prépare pas pleinement les jeunes à leur insertion professionnelle avec les visées sur le marché chinois tant sur le plan professionnel que sur le plan culturel et même linguistique. 


\section{Quel avenir pour le LEA dans la reconfiguration de l'enseignement supérieur ?}

Les mutations récentes de l'enseignement supérieur concernent directement les formations LEA. Il s'agit d'abord de l'internationalisation de l'enseignement supérieur dont les formes concrètes sont la mobilité des étudiants, les coopérations internationales et les diplômes conjoints avec des universités étrangères. Dans le cas de la Chine, de nombreux programmes de coopération se mettent en place dans le domaine des formations technologiques et scientifiques comme dans le domaine de l'économie et de la gestion. Se multiplient des formations en master, doctorat ou MBA, en partenariat avec des universités chinoises visant à former des spécialistes des affaires en Chine, aussi bien dans les écoles d'ingénieurs, les écoles de commerce que les universités. C'est donc en dehors des formations LEA et des départements d'études chinoises que se développent les formations professionnelles spécialisées sur la Chine. Dans une certaine mesure, ces formations universitaires de niveau master viennent concurrencer le LEA.

Des Instituts d'administration des entreprises (IAE), des facultés de droit et d'économie offrent une dizaine de formations spécialisées sur le monde chinois dans les domaines proches de la formation LEA : économie, droit et management. Ces spécialisations qui forment des cadres et responsables d'entreprises en gestion, commerce, affaires internationales ou relations interculturelles couvrent autant de domaines similaires aux masters LEA. Ces formations, souvent de master 2, suivent une démarche inverse de la formation LEA ; les étudiants sélectionnés ont tous suivi une formation initiale dans une spécialité : droit, économie, commerce ou science politique, etc. Les compétences en langue chinoise n'entrent pas en ligne de compte dans les critères de sélection. C'est pendant cette dernière année du master que les étudiants se spécialisent sur la Chine avec un enseignement intensif de langue suivi d'un séjour linguistique et d'un stage professionnel. Ces formations souvent encadrées par des professionnels et spécialistes de la Chine en partenariat avec des universités chinoises débouchent sur un diplôme mieux considéré que le diplôme de LEA par les milieux professionnels. 


\section{Shi Lu}

\section{Masters spécialisés sur la Chine dans les formations universitaires autres que le LEA ${ }^{14}$}

\begin{tabular}{|c|c|c|c|}
\hline Ville & Établissement & Titre du Master & Partenaires \\
\hline $\begin{array}{l}\text { Aix-en- } \\
\text { Provence }\end{array}$ & $\begin{array}{l}\text { Université de } \\
\text { Provence Aix- } \\
\text { Marseille 1, Institut } \\
\text { de recherches } \\
\text { Europe-Asie }\end{array}$ & $\begin{array}{l}\text { Systèmes de droit comparé : } \\
\text { monde chinois des affaires }\end{array}$ & \\
\hline $\begin{array}{l}\text { Aix-en- } \\
\text { Provence }\end{array}$ & $\begin{array}{l}\text { Université de } \\
\text { Provence Aix - } \\
\text { Marseille } 1\end{array}$ & $\begin{array}{l}\text { Négociation internationale } \\
\text { spécialité Extrême-Orient } \\
\text { (Chine, Corée, Japon et } \\
\text { Vietnam) }\end{array}$ & \\
\hline Angers & $\begin{array}{l}\text { Université d'Angers, } \\
\text { UFR Ingénierie du } \\
\text { tourisme }\end{array}$ & $\begin{array}{l}\text { Marchés émergents : monde } \\
\text { chinois }\end{array}$ & $\begin{array}{l}\text { Université Sun Yatsen, } \\
\text { Institut franco-chinois de } \\
\text { tourisme de l'université de } \\
\text { Canton, réseau du groupe } \\
\text { Accor, Garden Hotel }\end{array}$ \\
\hline $\begin{array}{l}\text { Clermont- } \\
\text { Ferrand }\end{array}$ & $\begin{array}{l}\text { Université } \\
\text { d'Auvergne, Faculté } \\
\text { économie et gestion }\end{array}$ & Économie de la Chine & \\
\hline Grenoble & $\begin{array}{l}\text { Université de } \\
\text { Grenoble, IAE }\end{array}$ & $\begin{array}{l}\text { MBA France-Chine, formation } \\
\text { délocalisée }\end{array}$ & Université de Shanghai \\
\hline Lyon & $\begin{array}{l}\text { Université Lyon 2, } \\
\text { IEP de Lyon }\end{array}$ & Affaires asiatiques & \\
\hline Nantes & $\begin{array}{l}\text { Université de } \\
\text { Nantes, IAE }\end{array}$ & $\begin{array}{l}\text { Management international } \\
\text { franco-chinois }\end{array}$ & \\
\hline Paris & $\begin{array}{l}\text { Université Paris 12, } \\
\text { Faculté } \\
\text { administration et } \\
\text { échanges } \\
\text { internationaux }\end{array}$ & $\begin{array}{l}\text { Commerce et affaires } \\
\text { internationales, options zone } \\
\text { Europe, zone Amériques, zone } \\
\text { Chine. }\end{array}$ & \\
\hline Paris & $\begin{array}{l}\text { Université Paris 1-la } \\
\text { Sorbonne, école de } \\
\text { droit }\end{array}$ & $\begin{array}{l}\text { Droit international et européen } \\
\text { des affaires, réservé à des } \\
\text { étudiants sinophones }\end{array}$ & $\begin{array}{l}\text { Université du peuple de } \\
\text { Pékin, université Tongji } \\
\text { de Shanghai }\end{array}$ \\
\hline Perpignan & $\begin{array}{l}\text { Université de } \\
\text { Perpignan }\end{array}$ & Management touristique chinois & Université de Suzhou \\
\hline Poitiers & $\begin{array}{l}\text { Université de } \\
\text { Poitiers, IAE }\end{array}$ & $\begin{array}{l}\text { Master administration des } \\
\text { entreprises, formation } \\
\text { délocalisée }\end{array}$ & $\begin{array}{l}\text { Université de Nanchang - } \\
\text { Institut franco-chinois de } \\
\text { Management }\end{array}$ \\
\hline
\end{tabular}

De fait, les entreprises connaissent encore mal la formation LEA et jugent insuffisant l'enseignement des disciplines professionnelles de cette filière. Certaines enquêtes montrent que les diplômés de la filière ont accès, lors de leur entrée sur le marché du travail, à des emplois sous qualifiés ${ }^{15}$. Les diplômés de master LEA se trouvent souvent dans des emplois

14 Source : sites des universités. Il s'agit d'un inventaire non exhaustif.

15 Richard de Vendeuil, «Langues étrangères : les bonnes filières », L'Express, 8 


\section{Les études chinoises et la professionnalisation}

intermédiaires alors qu'ils sont censés occuper un emploi à responsabilité - responsable import-export, chef de produits, cadre dans une organisation internationale etc. Selon l'enquête réalisée par l'université Lyon 3 en 2007 , les diplômés de master LEA - toutes langues confondues - sont majoritairement (un peu plus de $60 \%$ ) dans des professions intermédiaires. Ils occupent des emplois de type chef de projet dans l'événementiel, assistant import-export, assistant administratif ou attaché commercial et de gestion ${ }^{16}$. Seuls $29 \%$ d'entre eux occupent des emplois de catégorie cadre.

L'enseignement de langue dispensé dans les formations spécialisées citées ci-dessus invite à nous interroger sur la pédagogie. Longtemps, l'acquisition d'une langue étrangère a été considérée comme un travail de longue haleine. Ainsi l'organisation de l'enseignement, quelle que soit la filière (LEA ou LLCE), suit-elle un modèle classique distinguant la langue orale et la langue écrite. L'enseignement de la langue écrite occupe une place privilégiée en s'appuyant sur les cours qui nous semblent encore indispensables : grammaire, thème et version. Or, depuis quelques années, de nouvelles pratiques de l'enseignement de la langue viennent remettre en question ces acquis. Se fixant la communication pour objectif, ces nouvelles méthodes mettent en avant la pratique orale. Les écoles d'ingénieurs, les écoles de commerce et les formations universitaires spécialisées que nous venons d'envisager mettent en place un enseignement de la langue pragmatique, intensif, sur une courte période et suivi d'une immersion dans le pays de la langue étudiée.

En outre, l'apprentissage de plus en plus précoce des langues étrangères, dès l'école primaire pour certains, pourrait avoir un impact sur l'enseignement supérieur. Le chinois, considérée comme langue rare ou exotique il y a une dizaine d'années, est devenue la cinquième langue la plus enseignée, dans plus de quatre cents établissements secondaires en France aujourd'hui. À la rentrée de 2009, 25675 élèves se sont inscrits en chinois dans l'enseignement secondaire, contre seulement 9328 en $2004{ }^{17}$. Dans quelques années, le public étudiant sera très différent de celui d'aujourd'hui. Dé-

février 1996 ; SCUIO Lyon 3, opus cit.

16 SCUIO Lyon 3, opus cit., p. 7. Voir aussi Richard de Vendeuil, opus cit.

17 Marie-Estelle Puch, «Vif engouement pour l'enseignement du chinois », Le Figaro, 10 février 2010. 
sormais, la maîtrise de plusieurs langues étrangères chez les jeunes cadres est une nécessité. Les doubles diplômes, combinant la maîtrise de langues et une spécialisation, sortiraient des étudiants de l'impasse d'un cursus trop orienté vers les langues. L'objectif de la formation LEA, l'acquisition de deux langues étrangères, ne serait-t-il pas déjà déconnecté de la réalité ?

La professionnalisation est une donnée désormais bien intégrée dans l'enseignement supérieur. Dans la récente réforme du LMD, la formation professionnelle au même titre que la formation générale devient un choix d'orientation pour toutes les filières dès la licence. Le mariage entre le monde du travail et le monde universitaire est devenu une réalité incontestable. De ce point de vue, la formation LEA a su concilier les études de langues et un domaine professionnel pour s'adapter au nouveau contexte. La filière LLCE délaissée par des étudiants du fait des difficultés d'insertion professionnelle s'est réduite au profit de la filière LEA. Mais cela ne semble plus suffire aujourd'hui. Les nouveaux modes d'apprentissage des langues étrangères, les nouvelles méthodes d'enseignement et la concurrence des formations professionnelles universitaires nous invitent à réfléchir sur nos pratiques d'enseignement. Les enseignements spécifiques sur l'aire culturelle doivent être davantage pris en compte dans les maquettes d'enseignement du LEA. $\mathrm{Au}$ niveau de la licence, les enseignements sur la société chinoise (histoire, civilisation et économie, etc.) devraient être renforcés pour que les étudiants acquièrent des connaissances culturelles au même titre que les connaissances linguistiques. Quant au master LEA, les formations spécialisées sur le monde chinois paraissent insuffisantes, sinon peu visibles. Ne serait-il pas envisageable de créer quelques pôles de formation master LEA avec de vraies spécialisations bien ciblées sur le monde chinois, voire asiatique ? Nos enseignements en LEA comme en LLCE méritent d'être repensés pour trouver un nouveau souffle. 